\section{UK bill could prompt biodiversity loss}

The UK government's proposed Infrastructure Bill for England and Wales gives new powers to control or eradicate invasive, non-native species (see go.nature.com/kbkvtt). However, what constitutes such a species needs careful definition to ensure that any use of these powers is beneficial for conservation.

The draft bill defines nonnative species as those that are "not ordinarily resident in, or a regular visitor to, Great Britain". This definition covers past native species that are now extinct, species that may become naturally established under a changing climate, and species listed in Schedule 9 of the Wildlife and Countryside Act. That list contains native species, including some that have been reintroduced into the wild, such as the barn owl (Tyto alba), capercaillie (Tetrao urogallus), chough (Pyrrhocorax pyrrhocorax) and red kite (Milvus milvus). The legislation could also preclude future species reintroductions, a tool to counter biodiversity loss.

The current definition has serious implications for wildlife management. Once a species is classified as non-native, it can also be classified as invasive - and would therefore be subject to invasive-species legislation.

Proposed amendments to address these problems have been rejected. If the bill is passed in its present form, it could lead to an irreversible loss of native biodiversity.

Sarah Durant ${ }^{\star}$ Institute of Zoology, Zoological Society of London, UK. sarah.durant@ioz.ac.uk ${ }^{*}$ On behalf of 24 correspondents (see go.nature.com/9eembe for full list).

\section{Don't let microbial samples perish}

Microbial ecologists must coordinate to archive sample collections and genetic material. This will prevent valuable specimens from being lost to science and allow rigorous assessment of the effects of globally changing factors, disease and pollution on microbial communities.

Archiving is particularly valuable for hard-to-obtain or irreplaceable samples: for example, those from deep-sea hydrothermal vents or subglacial lakes in Antarctica. Samples may form an important time series, as in faecal material from extant populations never exposed to antibiotics, or soils from highlatitude systems threatened with biodiversity loss because of climate change.

Data generation is cheap, and getting cheaper. Technologies now exist to store DNA at room temperature for long periods and to reanalyse samples directly, which is better than trying to cobble together previous data sets generated using outdated methods. Reanalysis can also enable comparison of samples collected at different sites or time points.

In microbial ecology, proper sample archiving will accelerate advances, as collections of plants, animals and cultures have done for other areas of biology. Noah Fierer University of Colorado, Boulder, USA. Craig Cary University of Waikato, Hamilton, New Zealand. caryc@waikato.ac.nz

\section{Risk review is under way for invasive toad}

Sven Mecke and colleagues call for prior assessment of ecological risks that might be associated with eradication measures against the invasive Asian common toad Duttaphrynus melanostictus (Nature 511, 534; 2014). The Amphibian Specialist Group in Madagascar — part of the International Union for Conservation of Nature's Species Survival Commission network — is already undertaking such an evaluation, along with local and international experts.
We disagree that the effects of an invasive species need to be fully understood before starting control operations. Experience with other invaders shows that this could take decades, and swift action is crucial to stop an invasion from becoming widespread. We already know that $D$. melanostictus is invasive elsewhere in the tropics and is a biosecurity hazard in Australia.

Nationally coordinated by Christian Randrianantoandro, our efforts include determining the toads' distribution, providing educational materials to local communities and assembling experts to develop the feasibility study. We shall use genetic analyses to identify the source of introduction and will screen toads for pathogens and parasites. All toad sightings in Madagascar have so far been in urban and nearby degraded habitats, which would limit any threat to native biota should an eradication programme be carried out.

Costs of the preliminary assessment are estimated at US\$50,000. The Amphibian Survival Alliance is running an online fund-raising campaign, and we hope to involve international non-governmental organizations. A globally coordinated response may still stop the toads from invading Madagascar.

Franco Andreone ${ }^{*}$ Museo Regionale di Scienze Naturali, Turin, Italy.

franco.andreone@gmail.com ${ }^{*}$ On behalf of 11 correspondents (see go.nature.com/cdpbbq for full list).

\section{Ageing: develop models of frailty}

Good preclinical models of ageing are needed to discover the molecular mechanisms behind declining human physical performance (Nature 511, 405-407; 2014). The latest animal models of frailty are a step in the right direction.

For example, a genetically modified frail-mouse model mimics the inflammation and weakness that often afflicts older people (see A. Akki et al. Age 36, 21-30; 2014). Frailty can also be modelled in naturally ageing mice as a frailtyphenotype score, graded by such performance measures as grip strength and walking speed (H. Liu et al. J. Gerontol. A http://doi.org/t6p; 2013).

We and others have quantified a clinical frailty index in mice by tracking the accumulation of age-related deficits. This index increases with age in the same way as it does in humans (J. C. Whitehead et al. J. Gerontol. A 69, 621-632; 2014).

More-sophisticated animal models of frailty will need to include a broader range of performance measures if they are to properly represent the condition in people.

Susan E. Howlett, Kenneth Rockwood Dalhousie University, Halifax, Nova Scotia, Canada; and University of Manchester, UK. susan.howlett@dal.ca

\section{Ageing: research needs social science}

Translational biomedical research into ageing and longevity needs to include social science if it is to produce interventions for slowing physiological decline (Nature 511, 405-407; 2014).

Promoting a healthy lifespan depends on social factors as well as on medical insight. Any study on caloric restriction, for instance, should consider the cultural background of participants.

Social science also needs to be incorporated into therapeutic investigations - for example, to understand why some individuals do not take their medication. Such insight would boost compliance and therefore drug effectiveness.

Philipe de Souto Barreto Institute of Ageing, University Hospital of Toulouse (CHU Toulouse), France. philipebarreto81@yahoo.com.br 